\title{
Modelling drop-drop interactions in an atomic force microscope
}

\author{
Steven L. Carnie* Derek Y. C. Chan* \\ Rogério Manica*
}

(Received 27 October 2004)

\begin{abstract}
Recent experimental developments enabled dynamical measurement of the force between two moving oil drops in solution using an Atomic Force Microscope. The drop sizes (about 40 microns) and velocities (up to 15 microns/s) of the experiments produce a regime where surface forces, hydrodynamics and drop deformation are all significant. A detailed model of the experiments developed by Carnie, Chan, Dagastine, Lewis and Manica (2004) produces calculated force curves with attractive forces due mainly to hydrodynamic lubrication forces, in agreement with experiment. Details of the evolution of the drop surface deformations and surface velocity profiles are included in
\end{abstract}

${ }^{*}$ Particulate Fluids Processing Centre, Dept. of Mathematics and Statistics, The University of Melbourne, Parkville 3010, Australia.

mailto:S.Carnie@ms . unimelb. edu. au

See http://anziamj.austms.org.au/V46/CTAC2004/Carn for this article, (C) Austral. Mathematical Soc. 2005. Published September 1, 2005. ISSN 1446-8735 
movies accompanying this paper. A feature of the model is the use of a new boundary condition, obtained by matched asymptotic expansions, to incorporate the weak deformation at the drop scale into the thin film scale. This boundary condition is necessary to obtain results independent of the computational domain size. It also requires modeling assumptions about the three-phase contact line where the drops meet the solid surfaces of the Atomic Force Microscope. We determine the sensitivity of calculated force curves to two simple models for the three-phase contact line motion, and to the assumed values for the drop/solid contact angle.

\section{Contents}

1 Introduction

C806

2 The governing equations

C808

3 Results and discussion

C812

4 Conclusions

C817

References

C818

\section{Introduction}

The Atomic Force Microscope (AFM) has recently been used to make direct measurements of the forces involving deformable surfaces such as the interaction between a rigid probe particle and a deformable oil drop across an aqueous electrolyte solution [3, 4]. Dagastine et al. [5] measured the force between two approaching droplets of decane $(\approx 40 \mu \mathrm{m}$ radius $)$ in an aqueous solution of sodium dodecyl sulphate (SDS) in which one drop is attached 


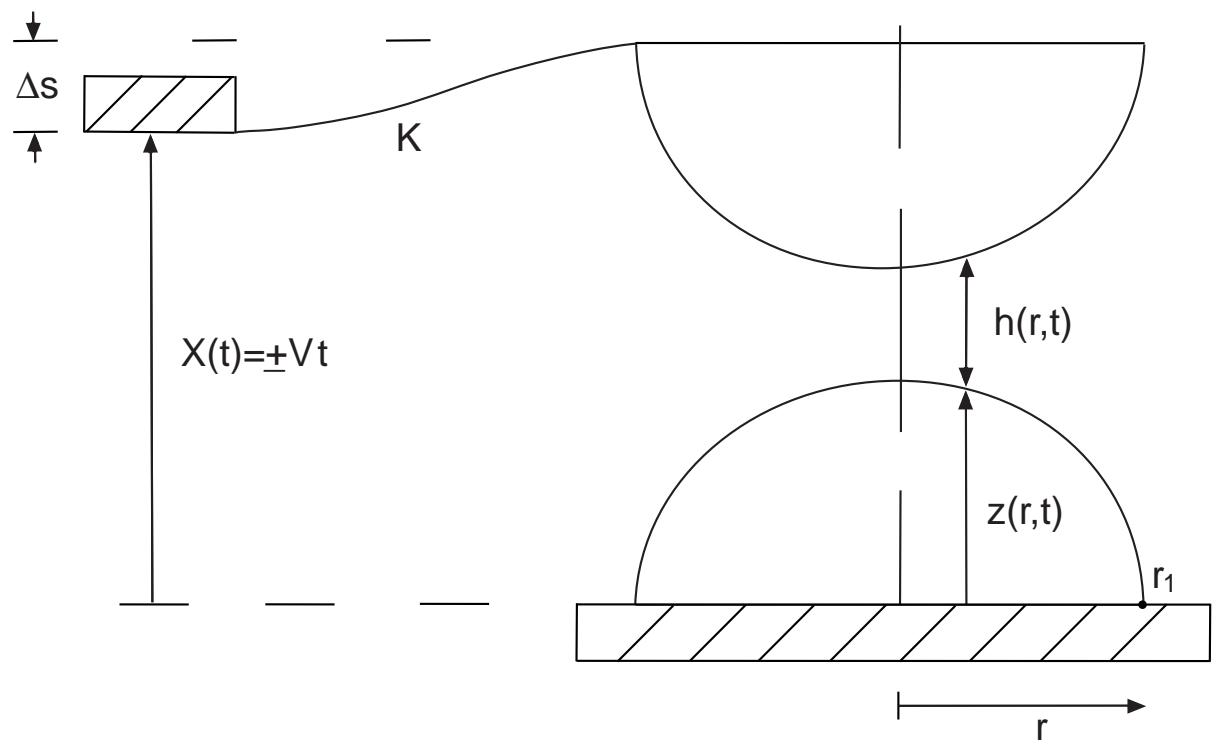

Figure 1: Schematic diagram of the AFM geometry of two interacting drops. $X$ measures the distance between the piezo stage and some fixed platform, $\Delta s$ is the cantilever deflection, $z(r, t)$ the drop height and $h(r, t)$ the film thickness.

to the AFM cantilever with known spring constant $K$. The other drop is attached to a piezoelectric stage that is moved according to a programmed velocity schedule. The stage is moved towards the cantilever with constant velocity $V$ over a displacement $\Delta X_{\max }$ and is then reversed over the same distance while the deflection of the spring is recorded and converted to a force $F$ using the spring constant of the cantilever. Therefore, the experimental data consist of values of the piezoelectric stage position $X$ and the corresponding force $F$.

A characteristic feature in the measured forces between decane drops [5] is the dependence on the approach velocity $V$. At low velocities, the force law 
is reversible, being the same on approach and retract, with an approximately constant compliance region. At higher velocities that are comparable to velocities due to thermal motion of drops in a suspension, the force curves show hysteresis. Attractive forces of significant magnitude are observed in the retract phase of the programmed velocity schedule.

Carnie et al. [2] modelled the AFM force measurement experiment between two drops in the experimental configuration of Dagastine et al. [5]. The results demonstrated the combined effects of electrical double layer repulsion between oil drops charged by adsorbed surfactant, hydrodynamic repulsion and attraction arising from drainage of the aqueous film between the drops and drop deformation due to surface forces, hydrodynamic forces and surface tension.

Here we undertake a systematic analysis of the consequences of the new boundary condition - the quantitative differences between two plausible boundary conditions of the three-phase contact line at the base of the drop:

1. having the three-phase contact line being held fixed while the contact angle changes to accommodate drop deformation, or

2. having the contact angle at the base of the drop being fixed with the position of the three-phase contact line changing during drop-drop interaction;

and the effect of the contact angle value in each case.

\section{The governing equations}

The problem divides naturally into two regions: the inner region where the drops interact, in which forces of interaction are important and the outer 
region, where the solution is obtained analytically. The equations are solved numerically in the inner region and the analytic solution in the outer region was used to develop a new boundary condition for the inner region.

If the interface contains significant amounts of adsorbed surfactant, there is much evidence to suggest that the interface is immobile and no tangential stress is transmitted into the drop [6, 7]. This means that the film velocity at the drop surface is the same as that of the drop interface and a no-slip boundary condition applies. Under these assumptions, together with axial symmetry and the usual thin lubricating film assumptions, the governing equation for the time evolution of the thickness of liquid film between two liquid drops is

$$
\frac{\partial h}{\partial t}=\frac{1}{12 \mu r} \frac{\partial}{\partial r}\left(r h^{3} \frac{\partial p}{\partial r}\right),
$$

where $h(r, t)$ is the film thickness as a function of radial coordinate $r$ and time $t, p(r, t)$ is the excess hydrodynamic pressure in the film relative to the bulk liquid, $\mu$ is the film viscosity (assumed Newtonian). This equation links the thinning rate of the film and the radial velocity due to a radial Poiseuille flow driven by a radial pressure gradient - for example, see [6] for derivations.

For the pressure, we use the Young-Laplace equation, which comes from a minimisation of the drop surface energy in the presence of external forces, subject to a constant volume (incompressibility) constraint:

$$
p+\Pi=\frac{2 \sigma}{R_{0}}-\frac{\sigma}{2 r} \frac{\partial}{\partial r}\left(r \frac{\partial h}{\partial r}\right),
$$

where $\sigma$ is the interfacial tension of the drop-film surface, $R_{0}$ is the harmonic mean $\left(R_{0}^{-1}=R_{1}^{-1}+R_{2}^{-1}\right)$ of the unperturbed radii of the two drops. $\left(2 \sigma / R_{0}\right)$ is the Lagrange multiplier associated with the constant drop volume constraint and $\Pi$ is the disjoining pressure. In the experiments of [5], a layer of adsorbed negatively charged surfactant on the drops produces a repulsive double layer disjoining pressure of the form $\Pi(h)=w \exp (-\kappa h(r, t))$ 
where $w=64 n k T \tanh ^{2}\left(e \psi_{0} / 4 k T\right)>0$ depends on the surface potential $\psi_{0}$ of the drops and $\kappa=\sqrt{2 n e^{2} / \epsilon k T}$ is the usual Debye-Hückel parameter that depends on salt concentration $n$.

In the inner region, the separation between undeformed surfaces, at an initial minimum separation $h_{0}$, is $h(r, 0)=h_{0}+r^{2} / R_{0}$. We assume the initial minimum separation $h_{0}$ is sufficiently large that surface forces are not important. Due to axial symmetry, we have the boundary conditions $\frac{\partial h}{\partial r}=0$ and $\frac{\partial p}{\partial r}=0$ at $r=0$ and at large radial distances the surfaces are so far apart that the film pressure approaches the bulk pressure, $p \rightarrow 0$ as $r \rightarrow \infty$.

In previous work [6] which modelled the approach of two spherical drops at constant velocity $V$ along the line of centres, the boundary condition $\dot{h}_{\text {rmax }}=\mp V$ was imposed at $r=r_{\max }$, the size of the computational domain. We show this boundary condition is inappropriate for the AFM experiment and produces a computed force sensitive to the choice of $r_{\max }$. Following previous work [1,3], Carnie et al. [2] derived the following new boundary conditions suitable for the situation of a drop sitting on a piezo stage which is moved at a set velocity $V$. The new boundary condition, which takes into account drop deformation during approach, has two forms:

$$
\dot{h}_{\mathrm{rmax}}+\dot{G}\left[2+\ln \left(\frac{r_{\max }^{2}}{4 R_{0}^{2}}\right)+\ln \left(\frac{1+\cos \theta}{1-\cos \theta}\right)-\frac{2 \pi \sigma}{K}\right]=\mp V,
$$

for the fixed (or stick) contact line case where $\theta$ is the contact angle of an undeformed drop at the piezo stage; and

$$
\dot{h}_{\mathrm{rmax}}+\dot{G}\left[2+\ln \left(\frac{r_{\max }^{2}}{4 R_{0}^{2}}\right)+\ln \left(\frac{1+\cos \theta_{p}}{1-\cos \theta_{p}}\right)-\frac{2}{2+\cos \theta_{p}}-\frac{2 \pi \sigma}{K}\right]=\mp V,
$$

for the case where the contact angle is fixed at $\theta_{p}$ while the three-phase contact line can slip during interaction. The quantity $G$ is related to the force $F$ between the drops by

$$
G=\frac{F}{2 \pi \sigma}=\frac{1}{\sigma} \int_{0}^{\infty} r[p(r, t)+\Pi(h(r, t))] d r .
$$


We use the following scales [6]

$$
h_{c}=R_{0} \mathrm{Ca}^{1 / 2}, \quad r_{c}=R_{0} \mathrm{Ca}^{1 / 4}, \quad p_{c}=\sigma / R_{0}, \quad t_{c}=\mu \mathrm{Ca}^{-1 / 2} / p_{c},
$$

where $\mathrm{Ca}=\mu V / \sigma$ is the capillary number (the ratio of viscous forces to surface tension forces) to produce the non-dimensional form of the equations, leading for example to the boundary conditions

$$
p=0 \quad \text { and } \quad \frac{\partial h}{\partial t}+\frac{d G}{d t} \mathcal{F}=\mp 1 \quad \text { at } r=r_{\max },
$$

where

$$
\mathcal{F}=\left[2+\ln \left(\frac{r_{\max }^{2} \mathrm{Ca}^{1 / 2}}{4}\right)+\ln \left(\frac{1+\cos \theta}{1-\cos \theta}\right)-\frac{2 \pi \sigma}{K}\right],
$$

if the position of the three-phase contact line of the drop is fixed. For the constant contact angle boundary condition $\theta=\theta_{p}$ (fixed)

$$
\mathcal{F}=\left[2+\ln \left(\frac{r_{\max }^{2} \mathrm{Ca}^{1 / 2}}{4}\right)+\ln \left(\frac{1+\cos \theta_{p}}{1-\cos \theta_{p}}\right)-\frac{2 \pi \sigma}{K}-\frac{2}{2+\cos \theta_{p}}\right] .
$$

We use central differencing in $r$ to obtain a system of differential equations for $h_{j}(t) \equiv h(j \Delta r, t), j=0, \ldots, N$ where $N=r_{\max } / \Delta r$. We use a uniform grid in $r=\left[0, r_{\max }\right]$ with $\Delta r=0.05$ and $r_{\max }=15$ producing a system of 300 equations. The boundary conditions at $r=0$ produce the equation for $\dot{h}_{0}$ and Eq. (7) provides the equation for $\dot{h}_{N}$. This requires $G$ as an extra variable to solve for. The functional $G$ is obtained by evaluating the following integral using Simpson's rule:

$$
G=\int_{0}^{r_{\max }} r[p(r, t)+\Pi] d r,
$$

which relates $G$ to all the other variables $h_{j}$ as an algebraic constraint. 
In summary, the final system of equations has the form

$$
\left(\begin{array}{ccccc}
1 & 0 & \cdots & 0 & 0 \\
0 & 1 & 0 & \cdots & 0 \\
& & \ddots & & \\
0 & 0 & \cdots & 1 & \mathcal{F} \\
0 & 0 & \cdots & 0 & 0
\end{array}\right)\left(\begin{array}{c}
\dot{h}_{0} \\
\dot{h}_{1} \\
\vdots \\
\dot{h}_{N} \\
\dot{G}
\end{array}\right)=\left(\begin{array}{c}
f_{0} \\
f_{1} \\
\vdots \\
\mp 1 \\
G-\sum_{j} w_{j} g\left(h_{j}\right)
\end{array}\right),
$$

where $\mathcal{F}$ is the coefficient of $\dot{G}$ in Eq. (7) and $f_{j}$ represent the discretized contributions of the thinning equation and normal stress balance. This system has a singular mass matrix and is a differential-algebraic equation (DAE) of index 1. It can be solved by standard software, in our case Matlab's ode15s.

\section{$3 \quad$ Results and discussion}

We present two movies to illustrate the deformation of the drop surface during approach and retract and the velocity of the drop surface. We also examine in detail: the sensitivity of the force between drops to the boundary condition; the effects of the behaviour at the three-phase contact line during interaction; and the influence of the contact angle at the three-phase contact line. The following parameters were used: identical drops with radius $R_{0}=40 \mu \mathrm{m}$, initial separation $h_{0}=0.7 \mu \mathrm{m}$, maximum piezo displacement $\Delta X_{\max }=0.9 \mu \mathrm{m}$, cantilever spring constant $K=0.028 \mathrm{~N} / \mathrm{m}$, concentration $9 \mathrm{mM}$ SDS, that gives a Debye length $\kappa^{-1}$ of $3.2 \mathrm{~nm}$ and a surface tension of about $8 \mathrm{mN} / \mathrm{m}$ [5]. These values are representative of those in the experiments [5].

In Figure 3(a) we show a movie of the surfaces of the two drops during the approach and retract phases at piezo stage velocities $V=13.2 \mu \mathrm{m} / \mathrm{s}(\approx$ twice the mean thermal velocity) and initial contact angle $\theta=90^{\circ}$, with a fixed contact line. As the drops approach they repel each other because they are 


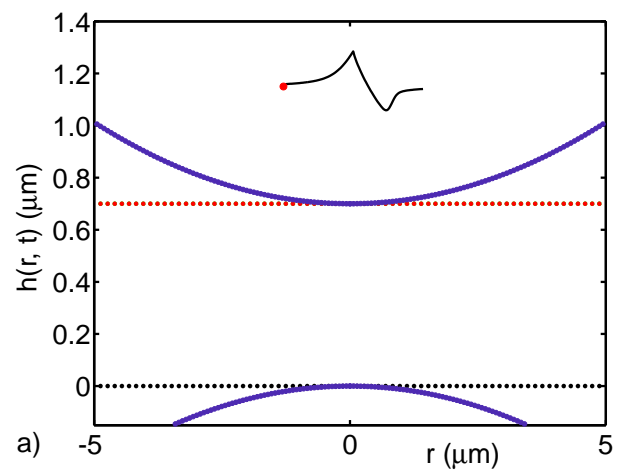

(a) Download the movie.

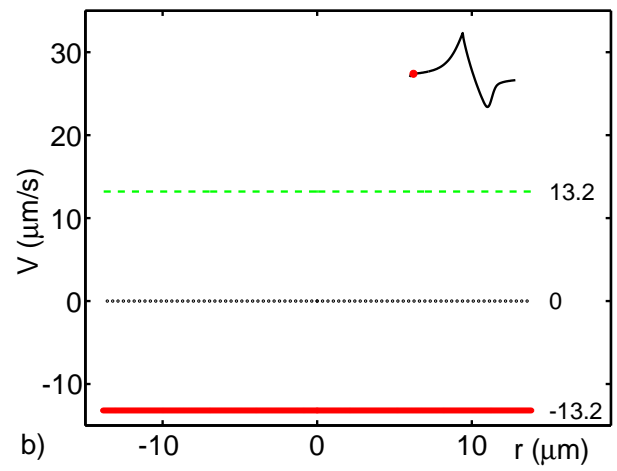

(b) Download the movie.

Figure 2: (a) Movie of interacting drops at piezo stage velocity $V=$ $13.2 \mu \mathrm{m} / \mathrm{s}$ with initial contact angle $\theta=90^{\circ}$ and the fixed contact line (Eq. (3)) boundary condition. The blue curves are the surfaces of the drops, the black line shows the motion of the piezo, the red line represents the movement of the cantilever and the green reference line indicates the initial position of the cantilever. The inset curve represents variations of the force with time, with the red dot showing the force for that time. (b) Movie of the relative velocity of the drop surfaces $\dot{h}$ or $V=13.2 \mu \mathrm{m} / \mathrm{s}, \theta=90^{\circ}$. The velocity is shown in red when the surfaces are approaching and in blue when they are separating. Reference lines for the initial and final velocities are shown in green.

equally charged and deform to create a thin film that increases in size in the radial direction. Regions of the surface in compression are indicated in red. During the retraction phase, regions of the surface that are in extension are shown in green.

In Figure 3(b) we show a movie of the relative velocity of the drop surfaces $\dot{h}(r, t)$. As the drops approach each other they feel the repulsive hydrodynamic and surface forces and the velocity decreases in the interaction region. At some point the central part of the film stops draining having reached its equilibrium position, while in the rest of the film the drops con- 
a)

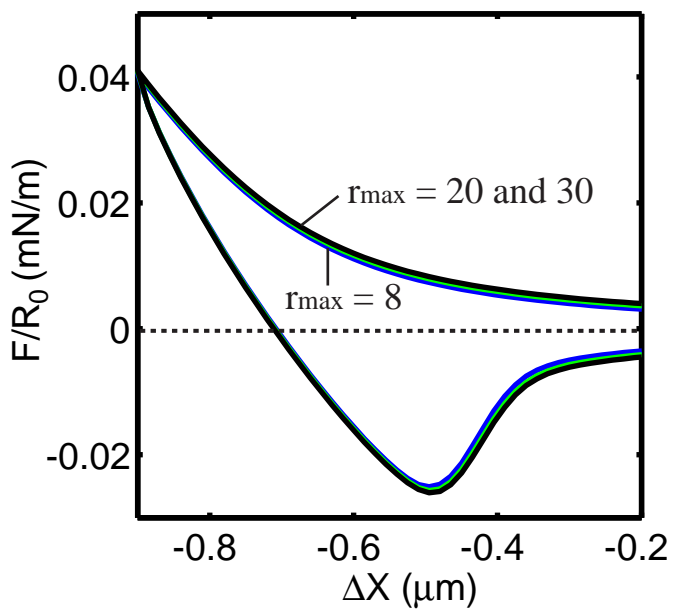

b)

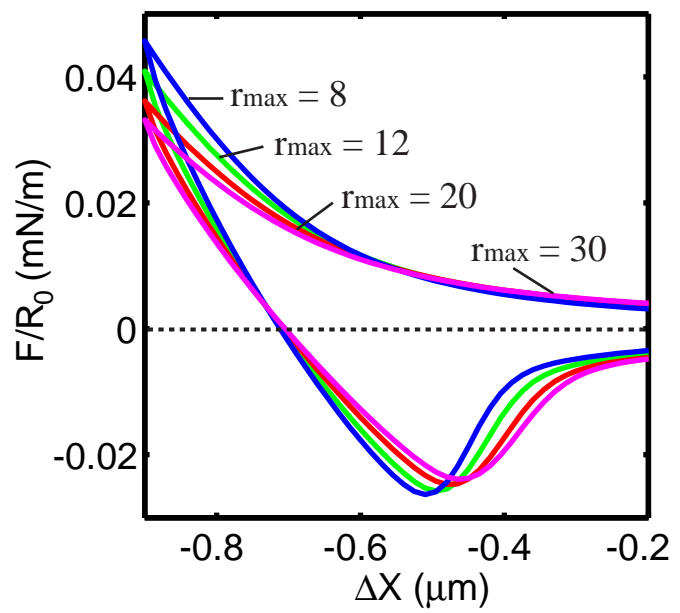

Figure 3: $F / R_{0}$ for $\theta=60^{\circ}, V=13.2 \mu \mathrm{m} / \mathrm{s}$ and various $r_{\max }$ (a) boundary condition Eq. (3) : $r_{\max }=8$ (blue), 12 (green), 20 and 30 (black) (b) boundary condition $\dot{h}_{\text {rmax }}=\mp V: r_{\max }=8$ (blue), 12 (green), 20 (red) and 30 (pink).

tinue their approach at a slowing rate. When retraction starts, the outer parts of each surface separate so the relative velocity suddenly becomes positive (indicated in blue) - however, the central part continues to approach. This central zone stays at constant thickness over the entire time when the force is attractive, a reflection of the attractive lubrication forces preventing drop separation. Eventually, the drops are pulled apart against lubrication forces and the central part separates faster to catch up with the outer region - this is depicted as a velocity overshoot in the movie. After that, the drops separate uniformly with velocity $V=13.2 \mu \mathrm{m} / \mathrm{s}$ as expected.

The results in Figure 3 show why our new boundary condition is necessary. Using the boundary condition $\dot{h}_{\text {rmax }}=\mp V$ (this condition is not correct for the AFM experiment), the force is sensitive to $r_{\max }$, the size of the compu- 
tational domain, see Figure 3(b). On the other hand, with the new boundary condition, see Figure 3(a), the computed force curves are independent of $r_{\max }$ for $r_{\max } \geq 15$.

The behaviour of the three-phase contact line when the drops are under a load depends on details of the drop-piezo stage interaction. Two limiting cases are where the three-phase contact line is held fixed (stick) or where the contact angle is fixed at $\theta_{p}$ (slip). In practice the three-phase line may stick/slip randomly many times during a force measurement. The two cases are compared in Figure 4(a) for two approach velocities. See that the force is smaller for the constant $\theta_{p}$ case (compare blue with green $(V=13.2 \mu \mathrm{m} / \mathrm{s}$ ) and red with pink $(V=2.4 \mu \mathrm{m} / \mathrm{s})$ ). This is because the drops have an extra degree of freedom when the contact line can slip - when under a load they can 'slip away' leading to a reduced force. This is most noticeable when the drops feel a strong repulsive force (they are pushed hard together); however, the force minimum is relatively insensitive.

The influence of the initial contact angle $\theta$ for the stick boundary condition is illustrated in Figure 4(b). Higher contact angles correspond to greater drop volumes - the initial radius of curvature is kept constant here but as the initial contact angle increases, the drops become a larger fraction of a sphere. For larger contact angles, the larger drops can 'back off' more, leading to thicker films and lower forces, both repulsive and attractive. A comparison with Figure 3(b) shows that the boundary condition $\dot{h}_{\mathrm{rmax}}=\mp V$ mimics the effect of increasing contact angle at fixed contact line - in effect, the drops are undeformable beyond $r=r_{\max }$. As $r_{\max }$ increases, the deformable volume of the drops increases, producing a reduced force at all separations. This continues without limit beyond reasonable values of $r_{\max }$. See a similar dependence on the contact angle $\theta_{p}$ for the slip boundary condition in Figure 4(c) and for the same reason.

Plots of the film profiles for the two boundary conditions and different values of $\theta, \theta_{p}$ in Figure 4(d) justify the remarks above. They show the final film thickness to be smallest for stick boundary condition and small contact 

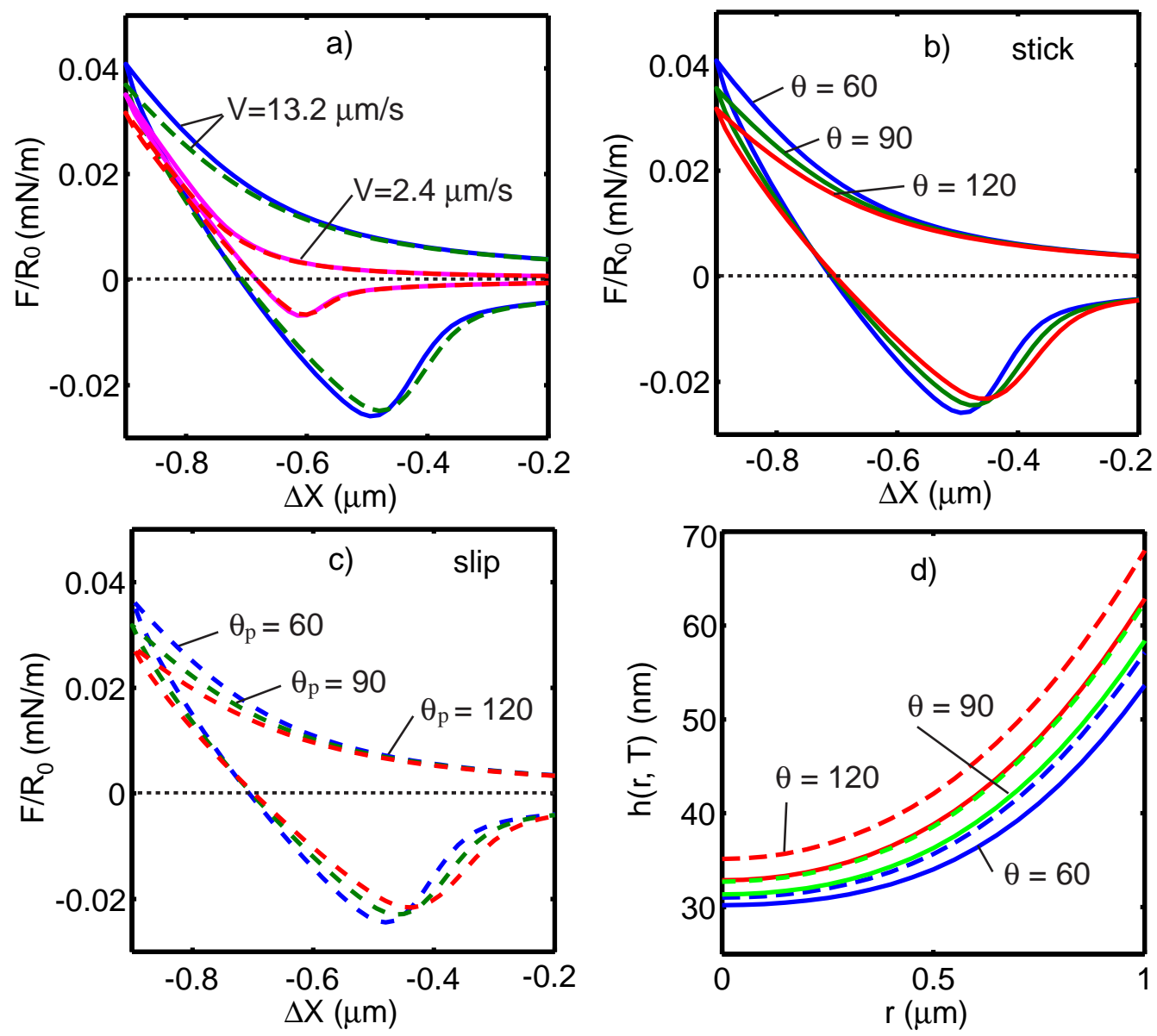

Figure 4: (a) $F / R_{0}$ for $\theta=60^{\circ}, V=13.2 \mu \mathrm{m} / \mathrm{s}$ : stick (blue) and slip (green) boundary conditions, $V=2.4 \mu \mathrm{m} / \mathrm{s}$ stick (pink) and slip (red); (b) $F / R_{0}$ for $V=13.2 \mu \mathrm{m} / \mathrm{s}$ and stick $\mathrm{BC}$ : $\theta=60^{\circ}$ (blue), $90^{\circ}$ (green) and $120^{\circ}$ (red); (c) $F / R_{0}$ for $V=13.2 \mu \mathrm{m} / \mathrm{s}$ and slip BC: $\theta=60^{\circ}$ (blue), $90^{\circ}$ (green) and $120^{\circ}$ (red); (d) film profiles at the force maximum for $V=13.2 \mu \mathrm{m} / \mathrm{s}$, stick (solid lines) and slip (broken lines) BC: $\theta=60^{\circ}$ (blue), $90^{\circ}$ (green) and $120^{\circ}$ (red). 
angles. In these cases the drops can back away from the load the least, the film is thinner and the force is higher.

\section{Conclusions}

This work describes new features of the interaction of two drops in an Atomic Force Microscope following the model of Carnie et al [2]. Two movies showing the deformation of the drops in an AFM experiment and the relative velocity of the drop surfaces give a vivid picture of the process.

Systematic comparisons show the importance of the new boundary condition in producing results independent of the computational domain size. Calculations with two plausible boundary conditions at the three-phase contact line show that the choice of boundary condition does not qualitatively alter the force curves but does have quantitative effects, especially when the force between drops is strongly repulsive. The force minimum is less sensitive.

The results with different boundary conditions and different contact angles are summarized as: when the drops have more freedom to minimize their interaction - either by slipping at the contact line or by having a larger volume (higher contact angle) - they do so. They approach less closely, leading to smaller forces of interaction, both repulsive and attractive.

Acknowledgment: This work was supported in part by a Discovery Project Grant of the Australian Research Council. The Particulate Fluids Processing Centre is an ARC Special Research Centre. RM is supported by a University of Melbourne International Research Scholarship. 


\section{References}

[1] D. C. Bardos. Contact angle dependence of solid probe-liquid drop forces in AFM measurements. Surface Science, vol. 517, pages 157-176, 2002. http://dx.doi.org/10.1016/S0039-6028(02)02054-X C810

[2] S. L. Carnie, D. Y. C. Chan, R. R Dagastine, C. Lewis and R. Manica. Measurement of dynamical forces between deformable drops using the Atomic Force Microscope I-Theory. Langmuir, submitted in 2004. http://dx.doi.org/10.1021/1a0475371 C808, C810, C817

[3] D. Y. C. Chan, R. R. Dagastine and L. R. White. Forces between a rigid probe and a liquid interface I. The repulsive case. J. Colloid Interface Sci., vol. 236, pages 141-154, 2001. http://dx.doi.org/10.1006/jcis.2000.7400 C806, C810

[4] R. R. Dagastine and L. R. White. Forces between a rigid probe and a liquid interface. J. Colloid Interface Sci. vol. 247, pages 310-320, 2002. http://dx.doi.org/10.1006/jcis.2001.8111 C806

[5] R. R. Dagastine, G. W. Stevens, D. Y. C. Chan and F. Grieser. Forces between two oil drops in aqueous solution measured by AFM J. Colloid Interface Sci., vol. 273, pages 339-342, 2004. http://dx.doi.org/10.1016/j.jcis.2003.11.001 C806, C807, C808, C809, C812

[6] E. Klaseboer, J. Ph. Chevaillier, C. Gourdon and O. Masbernat. Film drainage between colliding drops at constant approach velocity: experiments and modelling. J. Colloid Interface Sci. vol. 229, pages 274-285, 2000. http://dx.doi.org/10.1006/jcis.2000.6987 C809, C810, C811

[7] S. A. Nespolo, D. Y. C. Chan, F. Grieser, P. G. Hartley and G. W. Stevens. Forces between a rigid probe particle and a liquid interface: 
comparison between experiment and theory. Langmuir, vol. 19, pages 2124-2133, 2003. http://dx.doi.org/10.1021/la0260638 C809 